\title{
„Die Stärken von Oberwolfach sind die Transparenz der Entscheidungen und die Qualität der Workshops“
}

\author{
Thomas Vogt im Gespräch mit Gerhard Huisken
}

\begin{abstract}
Am 5. Juli 2019 feierte das Mathematische Forschungsinstitut in Oberwolfach sein 75-jähriges Bestehen mit einem Festakt. Aus diesem Anlass sprachen die DMV-Mitteilungen mit dem amtierenden Direktor Gerhard Huisken. In seinem Interview mit den Mitteilungen erzählt der 61-jährige über die turbulenten Anfangsjahre und den langsamen Aufstieg des Mathematischen Forschungsinstituts Oberwolfach in die Spitzenliga der Orte, an denen Mathematik-Geschichte geschrieben wird.
\end{abstract}

In welchem Geiste und mit welchen Zielen wurde das Mathematische Forschungsinstitut in Oberwolfach (MFO) vor 75 Jahren gegründet?

So wie ich die Geschichte verstehe, die ja auch untersucht worden ist - wir haben dazu ja mehrere Artikel auf unserer Webseite, zum Beispiel von Volker Remmert -, haben Mathematiker schon vor dem Krieg über ein mathematisches Forschungsinstitut nachgedacht. Während des Krieges, denke ich, dass die Naziherrschaft daran interessiert war, Kriegsforschung zu unterstützen und ein Reichsinstitut für Mathematik fördern wollte, weil sie sich davon Vorteile für Ihre Kriegsführung und für die Wirtschaft versprochen hat. Ob die damals handelnden Mathematiker dieselben Ziele hatten, weiß ich nicht; das lässt sich auch aus den Quellen nicht herauslesen, denn da steht ja nicht drin, was die wirklich gedacht haben. Aber dass die Durchsetzung dieses Institutes unter der Naziherrschaft nur möglich war, weil sich die Nazis davon Fortschritte im Krieg versprochen haben, das scheint mir ziemlich klar zu sein.

Welche Rolle spielte eigentlich Wilhelm Süß vor und nach dem Krieg?

Süß war Gründungsdirektor und er war politisch belastet. Auch das ist auf unserer Webseite dokumentiert. Süß war damals auch Rektor der Universität Freiburg und Präsident der Deutschen Mathematiker Vereinigung. Als DMV-Präsident hat er jüdische Mathematiker dazu gedrängt, die DMV zu verlassen. Nach dem Krieg wurde er von den französischen Besatzern als Direktor akzeptiert und hat es geschafft, trotz der Belastung aus der Nazizeit, das Institut an die internationale Forschung heranzuführen. Man muss die problematische Gründungszeit des Instituts klar benennen und auch weiter erforschen.

Wie ging es direkt nach dem Krieg weiter? Bitte skizzieren Sie kurz die wichtigsten Ereignisse seit Kriegsende.

Nach dem Krieg gab es sofort eine Umstellung ganz auf theoretische Mathematik, also möglichst weit weg von angewandter Mathematik oder gar Kriegsforschung. Entscheidend war nach dem Krieg vor allem, dass das Forschungsinstitut die ersten Jahre überhaupt überlebt hat, übrigens mit der Hilfe insbesondere ausländischer Mathematiker aus Frankreich und Großbritannien. Ein Beispiel dafür ist folgende heikle Situation:

Einmal stand das Schloss Oberwolfach, in dem sich damals das Forschungsinstitut befand, kurz vor der Besetzung durch französisch-marokkanische Soldaten. Nur dem beherzten Einschreiten des Mathematikers und britischen Offiziers John Todd war - aufgrund seiner Stellung - zu verdanken, dass die französischen Soldaten das Schloss nicht als Quartier beschlagnahmten. So konnte der Forschungsbetrieb weitergehen. Übrigens hat die Geschichte auch eine Fortsetzung: Später hat die Lebensgefährtin von John Todd, Rosemary Lonergan, der Oberwolfach Stiftung eine Spende zukommen lassen, so dass die Stiftung alle drei Jahre einen John Todd-Preis in numerischer Analysis vergeben kann. Auch war so im Jahr 2006 der Zukauf des umliegenden Geländes durch die GMF möglich, da die Bibliothekserweiterung über das alte Gelände ein Stück hinausging. Diesen Gebäudeflügel haben wir dann nach John Todd benannt, wo wir auch diese Geschichte erzählen.

Haben Sie noch ein weiteres Beispiel für die Fürsprache ausländischer Mathematiker für das MFO?

Berühmt ist zum Beispiel das Treffen 1949 mit Jean Dieudonné, Henri Cartan, Jean-Pierre Serre und René Thom. Gerade mithilfe dieser französischen Mathematiker und auch britischer Kollegen ist es gelungen, das Institut und damit die ganze deutsche Mathematik an die internationale Gemeinschaft heranzuführen. Ich denke, dass gerade diese positive Rolle des Instituts in den Nachkriegsjahren die Grundlage dafür war, dass es weiter finanziert und schließlich dauerhaft etabliert wurde. Ohne diese ersten positiven Jahre wäre das nicht gelungen.

Wie sah der Forschungsbetrieb damals aus?

Es gab damals nur ganz wenige Tagungen und es gab Einzelforscher. Zum Beispiel schrieb mir kürzlich Herr 
Roquette, wie er in Oberwolfach an seiner Dissertation gearbeitet hat und teilweise ganz alleine war und von dem Direktor Süß von Freiburg aus nur ein bis zweimal pro Woche in Oberwolfach besucht wurde. Der Betrieb war also noch sehr eingeschränkt. Eine der ersten Tagungen, die regelmäßig stattfanden, war die Geometrietagung von Reinhold Baer und von Süß.

Wie ging es nach dem Tod von Prof. Süß weiter?

Der Tod von Wilhelm Süß 1958 stellte einen großen Einschnitt dar. Süß hatte es immer wieder geschafft, die Finanzierung sicherzustellen. Diese Phase wird gerade erforscht in einem Forschungsprojekt von Volker Remmert. Dazu gibt es ganz aktuell einen Beitrag von Remmert in unserer Festschrift.

Nach dem Tod von Wilhelm Süß gab es zunächst eine Art Interregnum: Prof. Hellmuth Kneser war nur relativ kurz Direktor, von 1958 bis 1959. Ihm folgte Prof. Theodor Schneider von 1959 bis 1963. Er hat wichtige Strukturen geschaffen, zum Beispiel hat er wesentlich zur Gründung der „Gesellschaft für Mathematische Forschung“ (GMF) beigetragen.

Ende der Fünfzigerjahre war national und international schon so viel Vertrauen in das Institut vorhanden, dass dann der politische Wille da war, das Institut zu einer langfristigen Einrichtung zu machen. Dafür wurde im Juni 1959 die „Gesellschaft für Mathematische Forschung“ gegründet, um dem Vorhaben einen rechtlichen Rahmen zu geben.

Ab 1963 hat dann Direktor Barner die vorhandenen Strukturen genutzt und insbesondere mit Hilfe der VolkswagenStiftung die beiden großen Gebäude errichten lassen, die wir heute kennen: das Gästehaus und das Seminargebäude. Erst dadurch wurden regelmäßige Veranstaltungen mit 50 bis 60 Personen überhaupt möglich. In Folge hat Herr Barner den Forschungsbetrieb geprägt, wie wir ihn heute kennen, mit den regelmäßigen Seminaren und Workshops. Auch die Bibliothek wurde sorgfältig aufgebaut $\mathrm{zu}$ einer der besten in Mathematik weltweit.

Was war die nächste große Zäsur?

Das war sicherlich die Mitgliedschaft in der LeibnizGemeinschaft im Jahr 2005 zur Zeit meines Vorgängers Prof. Gert-Martin Greuel.

\section{Was hieß das konkret für das MFO?}

Damit war die Finanzierung des Instituts nicht mehr allein Ländersache. Die Hälfte der Mittel kommt seitdem vom Bund, die andere Hälfte gemeinsam von allen Bundesländern (nach dem Königsteiner Schlüssel). BadenWürttemberg hat aber als Sitzland weiterhin die Verwaltungshoheit. Damit war ein zentrales Finanzproblem gelöst: Zuvor gab es schwankend viele Mittel vom Land, aber auch aus ganz anderen Quellen. Volker Remmert hat jüngst herausgefunden, dass es zum Beispiel in den 1950er Jahren auch Zuwendungen vom Bundeskanzleramt gab. (lacht)

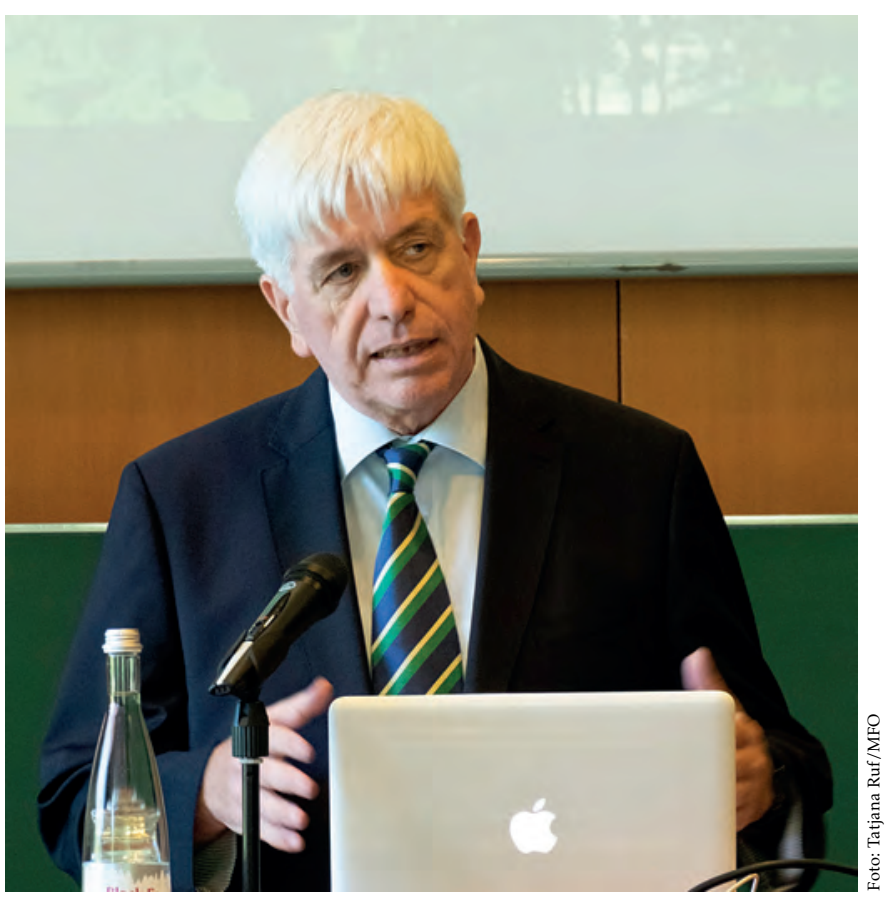

Gerhard Huisken spricht auf der Jubiläumsfeier des MFO

Durch die Mitgliedschaft in der Leibniz-Gemeinschaft war also die Grundfinanzierung des Instituts dauerhaft gesichert. Mit zusätzlichen Mitteln von Bund und Sitzland war es außerdem möglich, die in die Jahre gekommenen Gebäude zu sanieren. Mein Vorgänger Gert-Martin Greuel hat - zusammen mit dem damaligen Vorsitzenden der "Gesellschaft für mathematische Forschung“, Willi Jäger das gesamte Gästehaus rundum erneuern lassen und hat die Sanierung des Bibliotheks- und Hörsaalgebäudes in die Wege geleitet. Darauf konnte ich aufbauen und diese Gebäude mit Hilfe des Fördervereins, der Oberwolfach Stiftung und der Volkswagen Stiftung weiter modernisieren lassen. Mit Mitteln der Klaus Tschira Stiftung und der VolkswagenStiftung konnte der moderne Anbau des Bibliotheksgebäudes finanziert werden. Und neben einer neuen Heizungsanlage haben wir jetzt sogar eine Klimaanlage für die heißen Tage des Jahres, wie diese (lacht). Zusätzlich haben wir neue Diskussionsräume geschaffen und mehr Raum für Bücher und Zeitschriften.

Das heißt, Sie halten an den gedruckten Zeitschriften noch fest?

Ja, wir heben noch alles auf. Bei den Zeitschriften gehen wir vorsichtig über zu den elektronischen Zeitschriften, wenn wir sicheren Zugriff auf die Daten haben und die Langzeitarchivierung gesichert ist. Wir müssen einige der gedruckten Zeitschriften abbestellen, aber wir gehen dabei sehr behutsam vor. Und das wird ein Thema bleiben, also das sich Anpassen an die sich verändernde Publikationswelt ...

Und wie handhaben Sie es mit den Publikationen aus Oberwolfach?

Die Oberwolfach Reports mit den Abstracts aller hier gehaltenen Vorträge erscheinen in Kooperation mit dem 
Publishing House der European Mathematical Society sowohl in Hardcopy als auch als E-Journal. Sie können aber auch als einzelne pdf-Dateien in Open Access von unserer Webseite heruntergeladen werden. Wenn wir "Oberwolfach Seminare" haben, entstehen daraus manchmal Bücher bei Birkhäuser Science und dann entstehen noch die Preprints der individuellen Gäste, der Leibniz Fellows oder der „Research in pairs“. Und dann gibt es noch die so genannten Snapshots of Mathematics, die von Workshop-Teilnehmern geschrieben werden und sich an eine breitere Öffentlichkeit wenden. Die Snapshots bieten wir vornehmlich als pdf zum Herunterladen an und drucken wir nur manchmal als Sonderausgaben in kleiner Auflage. Übrigens haben wir das jetzt alles zusammengefasst in einer eigenen Publikationssektion auf unseren Internetseiten um open access, also den offenen Zugang, noch besser zu ermöglichen. Die Langzeitarchivierung realisieren wir zusammen mit der TIB Hannover.

Wie hat sich die inhaltlich Arbeit, die WorkshopSituation, im Laufe der Jahre gewandelt?

Also ein Trend, den aber schon mein Vorgänger beobachtet hat, ist, dass sich der Wettbewerb um die Workshops verstärkt hat. Auch Tagungen, die wiederholt unter demselben Namen stattfinden, und von denen es gar nicht mehr so viele gibt, zeigen einen starken Wechsel von Organisatoren, von Teilnehmern, von Schwerpunkten, die sich jeweils an die spezifischen Änderungen in den einzelnen Fächern anpassen. Und da achtet die Wissenschaftliche Kommission, die inzwischen 25 Mitglieder hat und sich laufend erneuert, sehr genau darauf, dass da keine verkrusteten Strukturen entstehen und ständig neue Themen aufgegriffen werden und hier in Oberwolfach wirklich die heißen Themen von den richtigen Leuten behandelt werden.

Wenn Sie dieses Wettbewerbsprinzip bitte einmal kurz erläutern könnten ...

Wer in Oberwolfach einen Workshop abhalten will, der muss einen Antrag einreichen. Die Deadline für einen Workshop im Jahr 2021 ist z. B. jetzt im Juli 2019. Der Antrag muss Angaben enthalten über das gewünschte Thema, den Ablauf und die einzuladenden Gäste. Die Anträge gehen dann an die Mitglieder der Wissenschaftlichen Kommission, die darüber Gutachten schreiben und sich dann treffen, - dieses Jahr im Oktober - zu einer Sitzung, auf der dann die endgültige Auswahl der über 40 Workshops im Jahr 2021 getroffen wird. Insofern kommt es auf die wissenschaftliche Qualität des Antrags, aber auch der Tagungsleiter an, welchem Antrag stattgegeben wird.

Bevorzugt die Wissenschaftliche Kommission theoretische Themen oder...

Nein, nein. Es gibt auch viele Workshops zu angewandten und interdisziplinären Themen, etwa zu mathematischphysikalischen oder mathematisch-chemischen Fragestellungen oder etwa zur Anwendung der Zahlentheorie in der Kryptographie. Und wir versuchen über die Mitglieder der Wissenschaftlichen Kommission alle wichtigen Bereiche abzudecken.

Sind Sie Mitglied in der Wissenschaftlichen Kommission?

Nein, ich organisiere den Begutachtungsprozess der Anträge und sage auch meine Meinung, aber ich habe kein Stimmrecht in der Kommission. Später lade ich dann nach Abgleich der Listen mit den Anträgen - die von den Tagungsleitern vorgeschlagenen Teilnehmer der Workshops ein. Der transparente und reibungslose Ablauf des Verfahrens ist von zentraler Bedeutung.

Welche Berechtigung hat das MFO heute und in $\mathrm{Zu}$ kunft in einer Zeit der Videokonferenzen, onlineJournals und Blogs?

Das Internet erlaubt natürlich den raschen Austausch von Ideen und prinzipiell die globale Verfügbarkeit von Publikationen. Aber das ist dann ja schon das Endprodukt. Ich denke, dass das Internet und die neuen Medien die persönliche Kommunikation, wie sie in Oberwolfach gelebt wird, in keinster Weise ersetzen können. In Oberwolfach entstehen am laufenden Band Ideen, die dann sofort diskutiert und manchmal auch sofort wieder verworfen werden, oft aber auch später zu großartigen Ideen und Lösungen beitragen.

Wodurch ist die Stärke von Oberwolfach noch definiert?

Ich glaube die große Stärke von Oberwolfach ist die kompetente und faire Arbeit der Wissenschaftlichen Kommission, also, dass jeder, der hier einen Antrag stellt, das Gefühl hat, gerecht beurteilt zu werden. Die qualitätvolle Arbeit der Kommission und der effiziente Ablauf der Tagungen sind eine wichtige Aufgabe des Instituts beziehungsweise der Institutsleitung. Oberwolfach steht für Qualität, Fairness und Effizienz, das ist in der community allgemein akzeptiert. Das ist der große Vorteil von Oberwolfach und das muss auch in Zukunft so bleiben!

Was ist noch für die Zukunft wichtig?

Was mir für die Zukunft noch wichtig ist, ist, dass wir sehr früh auf unsere Tagungen Nachwuchswissenschaftlerinnen und Nachwuchswissenschaftler einladen, die qualifiziert sind, um bei Ihnen früh die Begeisterung für das Institut zu wecken, wie bei den „gestandenen“ Wissenschaftlern/innen. Dabei müssen wir in Zukunft darauf achten bestehenden Ungleichgewichten entgegenzuwirken. Zum Beispiel ist die Anzahl der weiblichen Teilnehmer noch deutlich geringer als die der männlichen. Hier wollen wir bestehende Hindernisse abbauen und darauf achten, dass in Zukunft niemand vergessen wird.

Was bedeutet das konkret?

Wir müssen die Rahmenbedingungen weiter verbessern, also familienfreundlicher werden, zum Beispiel was die Kinderbetreuung anbelangt. Wir ermöglichen jetzt schon, dass Kinder und Angehörige zur Kinderbetreuung mitkommen und auch hier vor Ort mit dem Kind wohnen. 
Dafür stehen uns momentan drei Apartments zur Verfügung. Außerdem unterstützen wir bei der Suche nach Babysittern. Wir versuchen zu helfen, wo wir können und so den Anteil an Mathematikerinnen sukzessive zu steigern.

Bitte erläutern sie kurz die Programme, über die es Nachwuchswissenschaftlerinnen und Nachwuchswissenschaftlern jetzt schon möglich ist, an Workshops in Oberwolfach teilzunehmen.

Da gibt es verschiedene Förderprogramme, etwa die Oberwolfach-Seminare, die sich ganz direkt an den wissenschaftlichen Nachwuchs richten. Momentan finden sechs pro Jahr mit Unterstützung der Carl Friedrich von Siemens Stiftung statt. Die Leibniz Gemeinschaft hat die Programme „Oberwolfach Leibniz Fellows“ und „Oberwolfach Leibniz Graduate Students“ initiiert. Im ersten Programm können Post Docs bis zu fünf Jahre nach der Promotion bis zu drei Monate am Institut forschen, das zweite Programm ermöglicht Doktorandinnen und Doktoranden, an Workshops in Oberwolfach teilzunehmen. Und wir haben einen Grant der National Science Foundation in den USA, über den die Reisekosten von bis zu 1 oo Nachwuchswissenschaftlern pro Jahr gefördert werden.

Wie wollen Sie das Nachwuchsprogramm weiter ausbauen?

Momentan versuche ich Mittel zu bekommen, mit denen es Nachwuchswissenschaftlern auch aus anderen Regionen der Welt möglich wird, für Forschungsaufenthalte nach Oberwolfach zu kommen. Das wäre ein Ziel für die Zukunft, dass Nachwuchswissenschaftler, die eine Einladung bekommen haben, auch wirklich nach Oberwolfach kommen können.

\section{Welche Länder haben Sie da besonders im Blick?}

Das betrifft Länder, die momentan leider weniger vertreten sind, etwa in Afrika, Südamerika und Teilen Asiens.

Und worauf zielt das finanzielle Engagement der Simons Foundation?

Die Simons Foundation gibt uns zurzeit Geld für bis zu 40 "Simons Visiting Professors“. Das sind Wissenschaftler von außerhalb Europas - jung oder alt - , die einen Besuch in Oberwolfach mit einem Gastaufenthalt an einer anderen Forschungseinrichtung in Europa verbinden möchten. Das Programm zielt auf Synergien ab.

Sind Sie eigentlich bei vielen Workshops dabei?

Ich werde immer wieder mal zu Workshops offiziell als Tagungsteilnehmer eingeladen. An denen nehme ich, wenn es die Zeit erlaubt, ganz regulär teil. Bei den anderen Workshops, die mich interessieren, habe ich prinzipiell die Möglichkeit in Vorträge zu gehen. Das ist ein ganz besonderes Privileg des Direktors, von dem ich manchmal und dann sehr gerne Gebrauch mache.

\section{Haben Sie eigentlich noch Zeit für eigene Forschung?}

Das Klima in Oberwolfach ist so inspirierend und meine Mitarbeiterinnen und Mitarbeiter sind so routiniert, dass ich tatsächlich noch Zeit für eigene Forschungsarbeit habe und auch meine Doktorandinnen und Doktoranden an der Universität Tübingen betreuen kann, ja sicher.

Bitte sagen Sie uns zum Schluss noch kurz, was die Gäste der morgigen Jubiläumsfeier erwartet.

Morgen Nachmittag wird es zunächst eine Reihe von Grußworten geben. Zum Beispiel hat die Kultusministerin des Landes Baden-Württemberg, Theresia Bauer, ihr Kommen zugesagt; auch zugesagt hat Herr Dr. Meister, Staatssekretär im Bundesministerium für Bildung und Forschung. Cédric Villani wird leider nicht bei uns sein können, hat aber eine Videobotschaft geschickt. Fast alle Unterstützer unserer Arbeit werden das Wort an uns richten, also Vertreterinnen und Vertreter der Leibniz-Gemeinschaft, der Klaus Tschira Stiftung, der Oberwolfach Stiftung und der VolkswagenStiftung. Auch wird der Vorsitzende der Gesellschaft für mathematische Forschung, DMV-Präsident Götze, das Wort an uns richten. Stefan Müller, der das Institut seit vielen Jahren durch seine Mitarbeit in verschiedenen Funktionen und Gremien unterstützt hat und bestens kennt, wird den Festvortrag halten. Am Abend gibt es dann einen Sektempfang und ein Abendessen im Gasthaus zum Hirschen unten im Ort.

Dann wünsche ich Ihnen, wünsche ich uns, eine schöne Jubiläumsveranstaltung am morgigen Freitag.

Gerhard Huisken ist ein internationaler Spitzenforscher, der im Überschneidungsgebiet zwischen reiner Mathematik und theoretischer Physik arbeitet. Seine mathematischen Forschungsthemen sind die Analysis und die Differentialgeometrie; in der Physik hat er u. a. herausragende Beiträge zur allgemeinen Relativitätstheorie geleistet. Gerhard Huisken promovierte 1983 in Mathematik an der Universität Heidelberg. Drei Jahre später folgte die Habilitation. Von 1986 bis 1992 arbeitete er an der Universität Canberra in Australien, von 1992 bis 2002 war Huisken Professor an der Universität Tübingen, von 1999 bis 2000 Gastprofessor an der Princeton University. Von 2002 bis 2013 war Huisken Direktor am Albert-Einstein-Institut für Gravitationsphysik der Max-Planck-Gesellschaft in Golm bei Potsdam. Seit dem 1. April 2013 ist er Direktor des Mathematischen Forschungsinstituts Oberwolfach und Professor am Fachbereich Mathematik der Universität Tübingen. 